\title{
REVIEW
}

\section{Contemporary management of adult intramedullary spinal tumors - pathology and neurological outcomes related to surgical resection}

\author{
G Manzano, BA Green, S Vanni and AD Levi \\ Department of Neurological Surgery, University of Miami Miller School of Medicine, Miami, FL, USA
}

\begin{abstract}
Study Design: Retrospective chart review and review of the recent literature.
Objectives: To present our experience, over an 8-year period, with aggressive microsurgical resection of intramedullary spinal tumors in adults focusing on histology, microsurgical techniques, short-term neurological outcomes, complication avoidance and dorsal column dysfunction (DCD).

Setting: University of Miami/Jackson Memorial Medical Center Miami, FL, USA.

Methods: We conducted a retrospective review of a series of adult patients with intramedullary spinal tumors treated with microsurgical excision at the University of Miami/Jackson Memorial Medical Center between January 1997 and September 2005.

Results: Histologic analysis revealed a predominance of ependymomas (50\%) with astrocytomas only comprising $12.5 \%$ of the tumors. We found no significant difference in pre- and postoperative McCormick grades. Although patients did not manifest significant motor weakness postoperatively as a result of surgery, $43.6 \%$ of patients exhibited the signs and symptoms of DCD, resulting in significant postoperative morbidity.

Conclusion: We present a contemporary adult series of intramedullary spinal tumors. The most significant postoperative morbidity experienced by patients was DCD. The neurosurgeon should recognize the impact of these symptoms, prepare the patient and his/her family for the possibility of DCD, and minimize dorsal column manipulation in an attempt to decrease its prevalence.

Spinal Cord (2008) 46, 540-546; doi:10.1038/sc.2008.51; published online 10 June 2008
\end{abstract}

Keywords: dorsal column dysfunction; intramedullary tumor; ependymoma; astrocytoma

\section{Introduction}

The first successful removal of a spinal cord tumor dates back to 1887, through the cooperation of Gowers and Horsley. Diagnosis and preoperative planning, at that time, solely depended on a detailed neurological examination for precise localization of the lesion. ${ }^{1,2}$ In 1925, Charles Elsberg in his text on tumors of the spinal cord, recognized the advances made in diagnosing these tumors during his day, but conceded that 'the majority of tumors within the substance of the cord are infiltrating in character, and therefore not removable. ${ }^{3}$ Recognizing the surgical challenge posed by these lesions, neurosurgeons for decades thereafter, adopted a conservative approach consisting of biopsy and adjuvant radiotherapy. Recently, however, a more aggressive surgical approach has been advocated. ${ }^{4}$ Most notably, Epstein and his colleagues have challenged the traditional conservative

Correspondence: Dr AD Levi, Department of Neurological Surgery, University of Miami School of Medicine, Lois Pope Life Center, 1095 NW 14th Terrace, Miami, FL 33136, USA.

E-mail: alevi@med.miami.edu

Received 7 January 2008; revised 24 March 2008; accepted 22 April 2008; published online 10 June 2008 treatment paradigm and have published good results after aggressive resection of intramedullary tumors in both pediatric and adult patients. ${ }^{4-6}$ Our approach, for many years, has also been aggressive microsurgical resection. With recent technological advances in areas of neuroimaging, microsurgery and intraoperative neurophysiologic monitoring, aggressive resection of these lesions with acceptable perioperative morbidity has become a more realistic treatment goal.

Intramedullary spinal cord tumors comprise $4 \%$ of central nervous system tumors and $20 \%$ of all intraspinal tumors. ${ }^{5}$ The literature traditionally lists astrocytomas and ependymomas to be the most common histologic subtypes, and most series report that these occur with almost equal frequency in adults. ${ }^{7-10}$ In our experience, we found that astrocytomas constituted a very small percentage of intramedullary spinal tumors. We also found that the most debilitating postoperative morbidity experienced by these patients is dorsal column dysfunction DCD, a sequelae that has not been sufficiently defined or emphasized in the literature. We present our experience, over an 8-year period, 
with aggressive microsurgical resection of intramedullary spinal tumors in adults focusing on histology, microsurgical techniques, short-term neurological outcomes, complication avoidance and DCD.

\section{Materials and methods}

We conducted a retrospective review of a series of adult patients with intramedullary spinal tumors treated with microsurgical excision by the two senior authors (BAG and ADL) at the University of Miami/Jackson Memorial Medical Center between January 1997 and September 2005. Patients with filum terminale ependymomas and reactive/inflammatory lesions of the cord were excluded from the series. All other patients were included in the review, regardless of neurologic condition at time of presentation or prior treatment history.

We further analyzed, in detail, a subgroup of patients who had at least 3 months of follow-up. Only one patient in this subgroup had prior treatment for their intramedullary lesion. Clinical information was collected from inpatient hospital records and outpatient clinic records. Radiographic data was obtained from film review along with review of the neuroradiology reports. All histopathologic specimens were reviewed by attending neuropathologists at the University of Miami/Jackson Memorial Hospital. One specimen was sent to an outside center for a second opinion. In every case, the preoperative goal was complete microsurgical resection, as permitted by tumor margins and intraoperative electrophysiologic monitoring. In no case was the preoperative goal solely a biopsy. Patients' functional statuses were classified pre- and postoperatively using a Modified McCormick Scale (Table 1). Statistical analysis was carried out using analysis of variance to compare the effect of age, location of tumor, histology, extent of resection, use of hypothermia for neuroprotection and negative changes in intraoperative neurophysiologic monitoring on outcome as measured by McCormick grade.

\section{Results}

\section{Patient population}

Our series consisted of a total of 66 surgeries for intramedullary spinal tumors in a total of 64 patients. Two patients with a history of von Hippel-Lindau disease had two distinct operations for hemangioblastomas at different spinal levels. The age range was $17-81$ years, with a mean of 45 years, in 35 males and 29 females. Histologies consisted of 32 ependymomas (50\%), 10 hemangioblastomas (15.6\%), 8 astrocytomas $(12.5 \%), 6$ cavernomas $(9.4 \%)$ and 8 miscellaneous lesions (12.4\%), including 2 sarcoid lesions, 1 subependymoma, 1 epidermoid, 1 lipoma, 1 gliosarcoma, 1 melanoma and 1 cystic teratoma (Figure 1). Of the eight astrocytomas, three were pilocytic, four were low-grade and one was anaplastic. Thirty-four of the lesions were located in the cervical region $(51.5 \%), 17$ in the thoracic region $(25.6 \%), 8$ spanned the cervicothoracic junction (12.1\%), 4 spanned the cervicomedullary junction (6\%) and 3 spanned the thoracolumbar junction (4.5\%).
Table 1 Modified McCormick scale

\begin{tabular}{ll}
\hline Grade & Modified McCormick scale \\
\hline I & Intact neurologically, normal ambulation, minimal dyesthesia \\
II & Mild motor or sensory deficit, functional independence \\
III & $\begin{array}{l}\text { Moderate deficit, limitation of function, independent w/ } \\
\text { external aid }\end{array}$ \\
IV & $\begin{array}{l}\text { Severe motor or sensory deficit, limited function, dependent } \\
\text { V }\end{array}$ \\
\hline
\end{tabular}

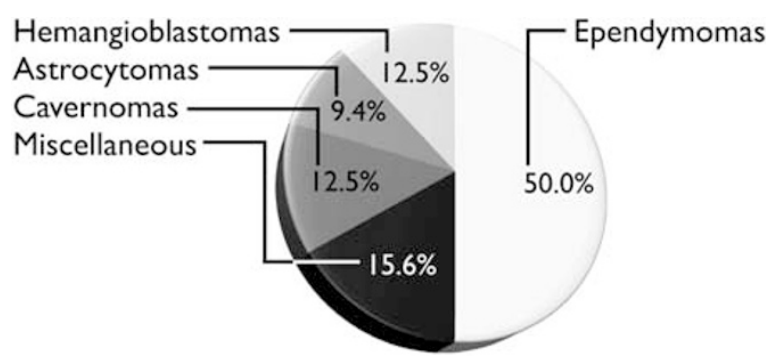

Figure 1 Relative incidence of intramedullary tumor histologies.

The subgroup of patients who were available for shortterm neurological outcome assessment consisted of 37 patients/39 operations with at least 3 months of follow-up. The age range for this group was $18-81$ years, with a mean of 44.8 years in 18 males and 19 females. The follow-up range was from 3 months to 6 years, with a mean follow-up of approximately 20 months. The histologic breakdown was relatively similar to that of the larger group, with 20 ependymomas (54\%), 6 hemangioblastomas (16.2\%), 4 astrocytomas $(10.8 \%), 3$ cavernomas $(8.1 \%)$ and 4 miscellaneous lesions $(10.8 \%)$ including 1 subependymoma, 1 lipoma, 1 gliosarcoma and 1 melanoma, and thus was felt to be representative of the larger group.

\section{MRI features}

Radiographically, 36 of the lesions (92.3\%) enhanced with gadolinium, with three of the lesions, a subependymoma, a low-grade astrocytoma and an anaplastic astrocytoma showing no enhancement. A syrinx was present in $80 \%$ of the ependymomas, $75 \%$ of the hemangioblastomas and $75 \%$ of the astrocytomas. Only one of the miscellaneous lesions, the melanoma had an associated syrinx. Nine $(23.1 \%)$ of the lesions exhibited hemorrhage of varied ages, including four ependymomas, three cavernomas, one hemangioblastoma and one melanoma.

\section{Surgical data}

Perioperatively, $28.2 \%$ of patients were treated with modest hypothermia and $33.3 \%$ were placed on a steroid protocol for neuroprotection. Approximately half of the patients (48.7\%) underwent a duraplasty for dural repair, and 35.9\% underwent prophylactic placement of a lumbar drain for postoperative cerebrospinal fluid diversion. Gross total resection was confirmed on postoperative imaging after $69.2 \%$ of the operations, whereas residual tumor on MRI 
(magnetic resonance imaging) was seen after $30.8 \%$ of the operations. The lesions amenable only to subtotal resection included five ependymomas, four astrocytomas, one melanoma, one cavernoma and one lipoma. In seven of these cases, the decision to limit the resection was influenced by a change in the patient's motor evoked potentials, (MEPs) during microsurgical resection.

\section{Preoperative clinical data}

The mean duration of symptoms was 25.3 months, with a range from several hours to 20 years. The predominant symptom at the time of presentation was sensory loss in the majority of patients (53.9\%), with 30.8\% complaining predominantly of variable combinations of a sensory level or sensory loss in an extremity, and $23.1 \%$ complaining primarily of gait difficulty, related to proprioceptive loss. A smaller number of patients complained predominantly of motor weakness $(20.5 \%)$, and pain $(20.5 \%)$, including neck pain and painful dysesthesias in the extremities. Two lesions (5.1\%) were found incidentally. Sphincter dysfunction, consisting of any combination of urinary frequency or incontinence, constipation or sexual dysfunction was reported in $30.8 \%$ of patients. The mean preoperative McCormick grade was 2.2, with 6 patients (15.4\%) presenting as a grade I, 24 patients (61.5\%) presenting as Grade II, 5 patients $(12.8 \%)$ presenting as grade III, 3 patients $(7.7 \%)$ presenting as grade IV, and 1 patient $(2.6 \%)$ presenting as grade $\mathrm{V}$.

\section{Postoperative clinical data}

The mean postoperative McCormick grade was 2.3 with 4 patients at grade I (10.3\%), 25 patients at grade II $(64.1 \%)$, 6 patients at grade III $(15.4 \%), 2$ patients at grade IV $(5.1 \%)$ and 2 patients at grade $5(5.1 \%)$. (Table 2 ) Fifty percent of the patients presenting with sphincter dysfunction showed improvement with respect to these symptoms, whereas the other half of these patients showed little change postoperatively at time of last follow-up. Two patients $(5.1 \%)$ manifested new sphincter dysfunction postoperatively. Time of last follow-up for these two patients was 3 months and their deficits consisted of urinary frequency and incontinence in one, and constipation in the other.

Seven patients (17.9\%) had a worsened postoperative McCormick grade. This group included four ependymomas, one hemangioblastoma, one gliosarcoma, and one anaplastic astrocytoma. The mean age for this group was 43.6 years. The mean duration of symptoms was 7.2 months and mean follow-up was 19.9 months. The mean preoperative McCormick grade for this group was 1.4, mean postoperative McCormick grade was 2.6. Three patients underwent gross total resections (two ependymomas and one hemangioblastoma), with the remainder undergoing subtotal resections. One patient with a T7-10 gliosarcoma initially presented as a McCormick grade III and underwent a subtotal resection. She remained a grade III after her first surgery, but represented several weeks later with acute onset paraplegia (grade V). She underwent a complete resection at that time and remained a grade $\mathrm{V}$ thereafter. Five patients went from a
Table 2 Preoperative and postoperative McCormick Grades

\begin{tabular}{ll}
\hline Preoperative McCormick & Postoperative McCormick \\
\hline 6 Grade I (15.4\%) & 4 Grade I $(10.3 \%)$ \\
24 Grade II $(61.5 \%)$ & 25 Grade II $(64.1 \%)$ \\
5 Grade III $(12.8 \%)$ & 6 Grade III $(15.4 \%)$ \\
3 Grade IV $(7.7 \%)$ & 2 Grade IV $(5.1 \%)$ \\
1 Grade V $(2.6 \%)$ & 2 Grade V $(5.1 \%)^{\mathrm{a}}$ \\
Mean $=2.2$ & Mean $=2.3$ \\
\hline
\end{tabular}

apatient re-presented after subtotal resection with tumor progression and paraplegia.

preoperative grade of I to a postoperative grade of II. One patient went from a preoperative grade of II to postoperative grade of III. Of this group of patients that exhibited deterioration in McCormick grade postoperatively, only one showed a decline in motor function and this was directly related to disease progression (gliosarcoma) and not to surgery. The remaining six patients manifesting a worsened McCormick grade postoperatively did so because of signs and symptoms related to dorsal column sensory dysfunction.

Four patients (10.3\%) showed improvement in their McCormick grade postoperatively. The histologies in this group included one ependymoma, one pilocytic astrocytoma, one hemangioblastoma and one cavernoma. The mean age for this group was 38.4 years and the mean length of follow-up was 12.8 months. The mean duration of symptoms was 28.3 months, with a mean preoperative McCormick grade of 2.5 and a mean postoperative McCormick grade of 1.5. One patient underwent a gross total resection (hemangioblastoma), with the rest undergoing subtotal resections. Three patients showed an improvement in McCormick grade from II preoperatively to an I postoperatively. One patient, with an ependymoma from C7-T1, presented with progressive spastic paraparesis and was unable to walk at the time of preoperative evaluation. He demonstrated an improvement in McCormick grade from a IV preoperatively to a III postoperatively, as he was ambulating well with the assistance of a cane.

\section{Statistical results}

No statistically significant correlation was found between age, location of tumor, histology, extent of resection, use of hypothermia for neuroprotection and negative changes in intraoperative neurophysiologic monitoring and outcome.

\section{Surgical complications}

There were two postoperative complications $(5.1 \%)$ in the group of 39 lesions with at least 3 months of follow-up. One was a wound dehiscence in the patient with the C7-T1 ependymoma who had undergone prior biopsy and radiotherapy. This was corrected with a wound debridement and repair with a local muscle flap. The second was a patient with a C2-3 ependymoma who developed a cerebrospinal fluid leak from their wound despite undergoing a duraplasty and being treated prophylactically with a lumbar drain. She was 
also found to have an Escherichia coli meningitis. She was treated with re-insertion of a lumbar drain and antibiotics and recovered fully. No patient developed postoperative spinal deformity during the follow-up period.

\section{Discussion}

Histology

Traditionally, ependymomas and astrocytomas are considered the two most common intramedullary spinal tumors. In adults, whereas it is generally believed that ependymomas outnumber astrocytomas, a review of the recent literature demonstrates that this is not always the case. Raco et al. ${ }^{7}$ in their large series $(n=202)$ of adult patients (mean age $=42.3$ years) found that astrocytomas (42\%) predominated, with ependymomas (34\%) following in frequency. ${ }^{7}$ Raco's series is an exception, and most series (Table 3$)^{2,5,7,11,12}$ show a predominance of ependymomas in adults, with astrocytomas generally thought to comprise roughly 30\% of adult intramedullary tumors. ${ }^{13,14}$ This is true even with the exclusion of filum terminale ependymomas. Our incidence of astrocytomas (12.5\%) is generally lower than that reported in the literature, as they ranked third in frequency behind ependymomas and hemangioblastomas in our series. The only series with a similarly low incidence of astrocytomas was that reported by Epstein's group. ${ }^{5}$ However, their series looked specifically at adult patients older than 50 (mean $=59.8$ years), whereas the mean age in our series was 45 years. The literature would, therefore, suggest that within adults as age increases, the incidence of ependymomas increases, whereas that of astrocytomas decreases.

In more recent adult series, intramedullary astrocytomas are becoming less and less prevalent. With improvements in MRI of the spinal cord, we can more readily distinguish between tumors and inflammatory/other pathologies preoperatively. Some 'astrocytomas' in older series of intramedullary 'tumors' may represent lesions which we would have either watched as low grade tumors or possibly represent small biopsies of inflammatory or demyelinating lesions erroneously labeled as astrocytomas. In the current MR era, we can get an excellent idea preoperatively as to the most likely histology. For example, it is known that intramedullary astrocytomas often exhibit minimal heterogeneous enhancement, have poorly demarcated margins and are generally not associated with a significant syrinx. Ependymomas, on the other hand, have more distinct margins radiographically, manifest more homogenous enhancement and generally have larger cystic cavities associated with them
(Figure 2). It has, therefore, become easier to distinguish these lesions radiographically and we are more likely to offer surgical resection in the latter scenario. Another possible explanation for the relative increased incidence of the ependymomas is our growing middle-aged and elderly populations, in which spinal cord astrocytomas are uncommon.

\section{Surgical strategy}

Surgical resection of any intramedullary tumor carries with it a significant potential risk of postoperative neurological worsening including but not limited to motor weakness, sensory loss and loss of autonomic function. Most modern series $^{4-7}$ advocate aggressive microsurgical resection of these lesions. With the aid of the operating microscope, neurophysiologic monitoring and intraoperative neuroprotection, complications can be minimized. As stated earlier, there were no statistically significant correlations found between negative changes in intraoperative neurophysiologic monitoring, and the use of hypothermia and outcome. With respect to neurophysiologic monitoring, outcome was not negatively affected because of our responsiveness to these changes intraoperatively. With the onset of any changes in the MEPs or somatosensory evoked potentials (SSEPs), our policy is to stop the resection, refrain from any cord retraction and allow the cord to 'rest' for several minutes. After ensuring that the signals are not being affected by hypotension, depth of anesthesia or temperature, we reassess the monitoring parameters, and continue only if these have returned to baseline. Persistent, negative trends in the monitoring
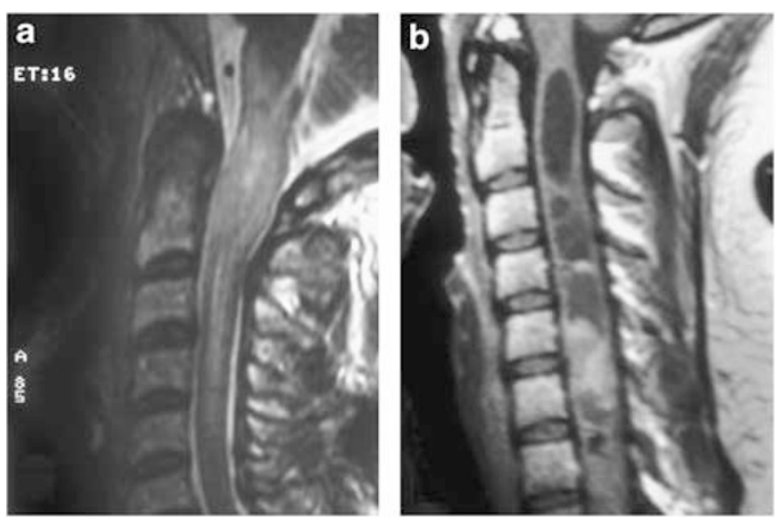

Figure 2 Imaging characteristics. (a) T2 images of infiltrating astrocytoma with poorly defined margins and significant cord edema. (b) T1 images with gadolinium of ependymoma with clear enhancement, discernable margins and a large associated syrinx.

Table 3 Relative incidence of adult intramedullary ependymomas and astrocytomas tumors in the recent literature

\begin{tabular}{|c|c|c|c|c|}
\hline Authors & Series (n ) & Age (years) & Ependymomas (\%) & Astrocytomas (\%) \\
\hline Miller ${ }^{12}$ & 177 & All > 21 & 53 & 27 \\
\hline Sandalcioglu et al. ${ }^{11}$ & 78 & Mean 43 & 41 & 19 \\
\hline Raco et al. ${ }^{7}$ & 202 & Mean 42 & 34 & 42 \\
\hline Regelsberger et al. ${ }^{2}$ & 78 & Mean 52 & 63 & 18 \\
\hline Shrivastava et al. ${ }^{5}$ & 30 & Mean 60 & 83 & 10 \\
\hline
\end{tabular}


parameters generally prompt us to limit the surgery to a subtotal resection. Hypothermia is a more recent adjunct that has been employed with only $28.2 \%$ receiving modest hypothermia. The goal of prophylactic modest hypothermia is used to limit the primary and secondary physiologic mechanisms that lead to neuronal injury after spinal cord manipulation and tumor resection.

Given the relatively indolent nature of many of these tumors, in cases where there are poorly defined planes between the lesion and the spinal cord, safe surgical decision-making should be the rule. Although there is a role for radiotherapy, particularly in more histologically aggressive tumors, ${ }^{15-17}$ the natural history of the majority of these lesions is generally benign, and thus radiation is clearly not a first or even secondary line of treatment. Its associated side effects, including neurologic worsening from tissue edema or radiation necrosis and the potential wound healing complications after initial and subsequent microsurgical resections, cannot be ignored. The role of focused radiotherapy such as cyberknife radiosurgery is yet to be determined.

In keeping with the general principles of surgical oncology, our philosophy has been to completely resect these lesions to afford patients the greatest chance at disease-free survival while at the same time, eliminating the threat of progressive neurologic decline from tumor progression. We also hope to eliminate the need for adjunctive radiotherapy due to its associated potential complications. Our preoperative planning and intraoperative decision-making take full advantage of technological advances in the fields of neuroimaging, microsurgery and neurophysiologic monitoring. Preoperative MRI sequences with and without gadolinium are of the utmost importance in delineating the position of the lesion within the spinal cord, and identifying associated cysts, syrinxes and hemorrhage. After dural exposure, intraoperative ultrasound is used to ensure adequate rostrocaudal exposure of the tumor. Once the cord is exposed, the dorsal median sulcus is located by following the course of penetrating vessels arising from the mid-line dorsal medullary vein, and the myelotomy is centered over the sulcus. In cases where the lesion is laterally situated in the cord, an approach through the dorsal root entry zone is employed. Using microsurgical-dissecting tools we gently develop a plane circumferentially around the lesion. The Orion-1 tumor desiccator (Ortho Development, Salt Lake City) or cavitron ultrasonic surgical aspirator (CUSA) (Integra LifeSciences, Plainsboro) is also used to debulk the lesion to minimize traction on the spinal cord during resection, making the process less traumatic to the surrounding cord parenchyma. Motor-evoked potential and somatosensoryevoked potentials are used throughout the resection process. We do not use pial sutures during tumor exposure or after tumor resection. We believe that retracting the dorsal columns with pial sutures can potentially increase the incidence and severity of DSD. We also believe that closing the pia over the tumor resection cavity can theoretically promote the development of a postoperative syrinx.

In selected cases a number of perioperative adjuncts for neuroprotection have been used including the use of methylprednisolone and modest hypothermia. Patients are given with a loading dose of solumedrol according to the NASCIS II protocol. Modest hypothermia is induced via an Alsius central venous cooling catheter (Alsius, Irvine) that is placed into the femoral vein prior to positioning the patient prone for surgery. The patient's temperature is reduced to 33.5 degrees Celsius, and is maintained throughout the case. The patient is slowly re-warmed to normal body temperature at variable rates over the course of $48 \mathrm{~h}$ postoperatively, depending on their postoperative neurologic status. Perioperative steroids are reserved for cases demonstrating neurologic deterioration attributable to spinal cord edema and or manipulation. There was no statistically significant difference in outcomes with the use of these measures as determined by McCormick grade in the subset of patients evaluated.

A post-resection expansile duraplasty with cadaveric dural allograft and prophylactic postoperative cerebrospinal fluid diversion with a lumbar drain were used selectively in this surgical series as measures to widen the subarachnoid compartment and decrease the risk of postoperative cerebrospinal fluid leak, respectively. No significant relationship was found between these measures and the two occurrences of postoperative wound complications. In the patient with a wound dehiscence, this was probably related to poor soft tissue and skin quality from prior radiation therapy. The other wound, with a cerebrospinal fluid leak and meningitis developed despite the use of an expansile duraplasty and a prophylactic cerebrospinal fluid drain.

\section{Dorsal column dysfunction}

For decades it has been recognized that the overwhelming risk in surgically treating intramedullary lesions is rendering a patient plegic as a result of cord manipulation. This limiting factor has become a less prevalent complication, as improved microsurgical techniques and intraoperative neurophysiologic monitoring have allowed the neurosurgeon to successfully resect these lesions with a minimal incidence of postoperative motor deficits.

Not enough importance has been given, in the literature, to the debilitating postoperative DCD experienced by these patients. The myriad of symptoms which fall under this category include significant generalized numbness or painful dysesthesias below the surgical level and proprioceptive loss with gait dysfunction (Figure 3). In addition to the obvious impact that gait dysfunction and painful dysesthesias has on a patient, the generalized numbness is also extremely disturbing and incapacitating in our experience.

The general surgical approach to these lesions is through a midline myelotomy, between the dorsal columns, with subsequent gentle retraction of the dorsal columns during tumor resection. It is not uncommon, during this stage, to see changes in the sensory evoked potentials, including decreased amplitude and increased latencies. Dorsal column manipulation is an inherent part of the surgical approach and DCD is a prevalent postoperative morbidity. New or increased DCD was seen in $43.6 \%$ of our patients. Of the seven patients with a worsened McCormick postoperatively in our series, DCD was the causative factor in six of the patients. 
a

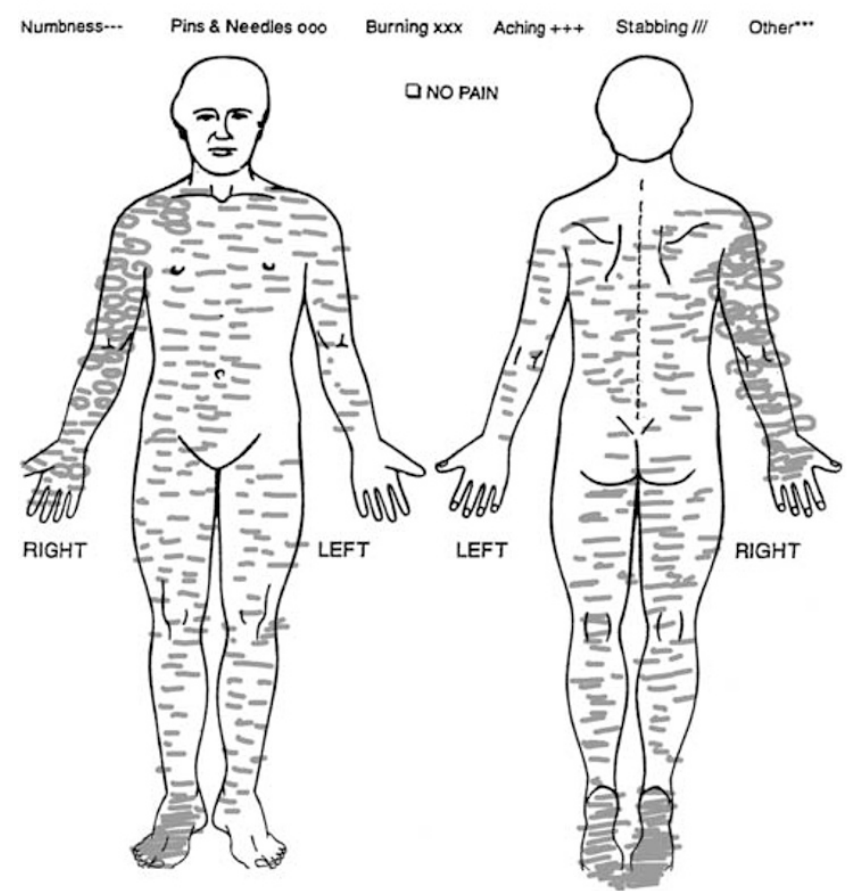

b

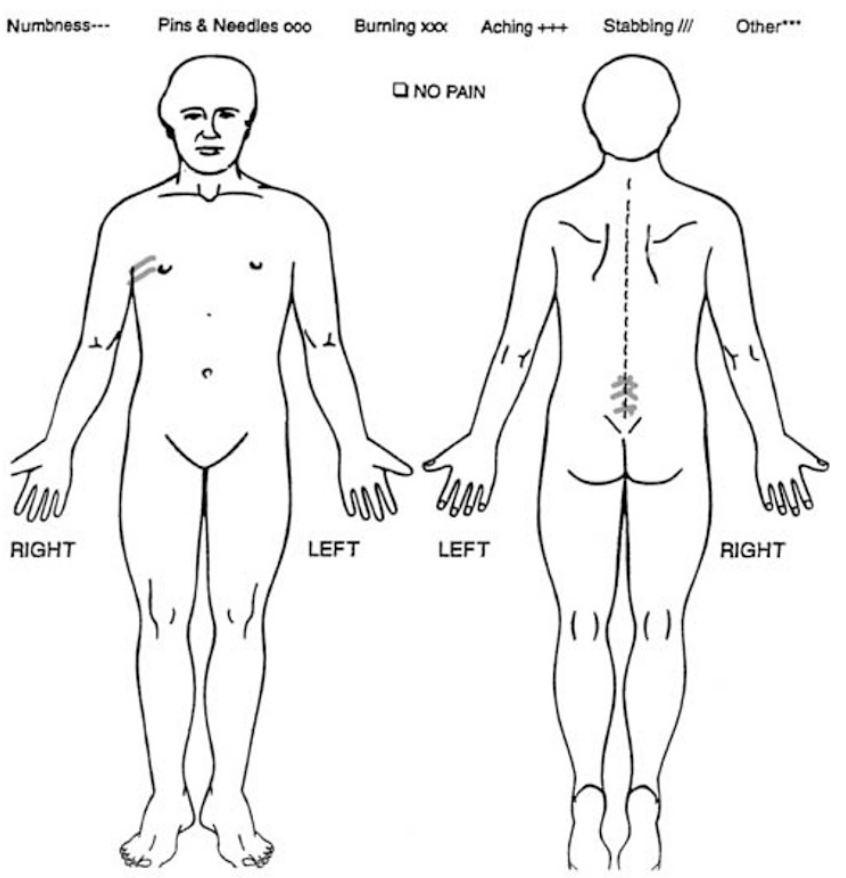

Figure 3 Self-reported postoperative pain drawing for two different patients demonstrating variable degrees of dorsal column dysfunction. It is our impression that the degree of postoperative dorsal column dysfunction is affected by the location of the intramedullary lesion and therefore, the cord manipulation/retraction needed for excision. (a) Patient with significant numbness and dysesthesias after resection of cervical ependymoma. (b) Patient with essentially no sensory deficits after resection of thoracic ependymoma.

The morbidity of DCD must be seriously considered when deciding if surgery, versus observation is the appropriate treatment option for a patient. An older patient with mild symptoms and a slow growing tumor may be better served with observation, rather than subjecting them to significant DCD postoperatively. If surgery is the right option, then a detailed explanation of the possibility of postoperative DCD should be given to the patient and their families to allow them to prepare emotionally, psychologically and practically for this condition.

In addition to preparing the patient for the possibility of DCD, the surgeon should always strive to refine his/her surgical technique, to minimize dorsal column damage. This is especially true with deeper intramedullary lesions that require greater dorsal column manipulation for resection. A high-resolution microscope is essential in attempting to identify the dorsal midline sulcus, to minimize the damage to adjacent white matter tracts, and to develop a plane within the sulcus. The exact location of the sulcus is facilitated by identifying small veins, which arise from the mid-line dorsal vein and penetrate the pial surface at right angles. Pial sutures with gentle, steady retraction of the dorsal columns may also be a good alternative to continuous manipulation throughout the tumor resection. We also try to limit the extent of the dorsal column incision particularly at the rostral and caudal poles of the tumor, because as the tumor tapers, more white matter manipulation will be required. Instead we try to angle the microscope to visualize the tapered ends of the tumor working through the central area of the myelotomy - that is, an area where the tumor is closest to the pial surface. The anatomy of the tumor and its relationship to the pial surface and sulcus are critical determinants in assessing the risk of DCD.

\section{Summary}

We present a contemporary adult series of intramedullary spinal tumors. Histologic analysis revealed a predominance of ependymomas (50\%) with astrocytomas only comprising $12.5 \%$ of the tumors. We found no significant difference in pre- and postoperative McCormick grades. Although patients did not manifest significant motor weakness postoperatively as a result of surgery, $43.6 \%$ of patients exhibited the signs and symptoms of DCD, resulting in significant postoperative morbidity. The neurosurgeon should recognize the impact of these symptoms, prepare the patient and his/her family for the possibility of DCD and minimize dorsal column manipulation in an attempt to decrease its prevalence.

\section{References}

1 Toellner R. Illustrierte geschichte der medizin. Weltbild Verlag: Augsburg, 2000.

2 Regelsberger J, Fritzsche E, Langer N, Westphal M. Intraoperative sonography of intra- and extramedullary tumors. Ultrasound in Med Biol 2005; 31: 593-598.

3 Elsberg C. Tumors of the Spinal Cord and the Symptoms of Irritation and Compression of the Spinal Cord and Nerve Roots: Pathology, Symptomatology, Diagnosis and Treatment. Hoeber: New York, 1925. 
4 Cooper P, Epstein F. Radical resection of intramedullary spinal cord tumors in adults. Recent experience in 29 patients. J Neurosurg 1985; 63: 492-499.

5 Shrivastava R, Epstein F, Perin N, Post K, Gallo G. Intramedullary spinal cord tumors in patients older than 50 years of age: management and outcome analysis. J Neurosurg Spine 2005; 2: 249-255.

6 Constantini S, Miller D, Allen J, Rorke L, Freed D, Epstein F. Radical excision of intramedullary spinal cord tumors: surgical morbidity and long-term follow-up evaluation in 164 children and young adults.. J Neurosurg 2000; 93 (2 Suppl): 183-193.

7 Raco A, Esposito V, Lenzi J, Piccirilli M, Delfini R, Cantore G. Long-term follow-up of intramedullary spinal cord tumors: a series of 202 cases. Neurosurgery 2005; 56: 972-981.

8 Chang U, Choe W, Chung S, Kim H. Surgical outcome and prognostic factors of intramedullary ependymomas in adults. I Neurooncl 2002; 57: 133-139.

9 Ferrante L, Mastronardi L, Celli P, Lunardi P, Acqui M, Fortuna A. Intramedullary spinal cord ependymomas: a study of 45 cases with long-term follow-up. Acta Neurochir (Wien) 1992; 119: 74-79.

10 Kane P, el-Madhdy W, Singh A, Powell M, Crockard H. Spinal intradural tumours: Part II-Intramedullary. Br J Neurosurg 1999; 13: $558-563$.
11 Sandalcioglu I, Gasser T, Asgari S, Lazorisak A, Engelhorn T, Egelhof $\mathrm{T}$ et al. Functional outcome after surgical treatment of intramedullary spinal cord tumors: experience with 78 patients. Spinal Cord 2005; 43: 34-41.

12 Miller D. Surgical pathology of intramedullary spinal cord neoplasms. J Neurooncol 2000; 47: 189-194.

13 Koeller K, Rosenblum S, Morrison A. Neoplasms of the spinal cord and filum terminale: radiologic-pathologic correlation. AFIP archives 2000; 20: 1721-1749.

14 Baleriaux D. Spinal cord tumors. Eur Radiol 1999; 9: 1252-1258.

15 McLaughlin MP, Marcus Jr RB, Buatti JM, McCollough WM, Mickle JP, Kedar A et al. Ependymoma: results, prognostic factors and treatment recommendations. Int J Radiation Onc Biol Phys 1998; 40: 845-850.

16 McLaughlin M, Nuati J, Marcus Jr R, Maria B, Mickle P, Kedar A. Outcome after radiotherapy of primary spinal cord glial tumors. Radiat Oncol Invest 1998; 6: 276-280.

17 Abdel-Wahab M, Corn B, Wolfson A, Raub W, Gaspar LE, Curran $\mathrm{Jr}$ W et al. Prognostic factors and survival in patients with spinal cord gliomas after radiation therapy. Am J Clinical Oncol 1999; 22: 344-351. 